Modeling, Identification and Control, Vol. 39, No. 1, 2018, pp. 15-21, ISSN 1890-1328

\title{
Parameter-dependent PWQ Lyapunov function stability criteria for uncertain piecewise linear systems
}

\begin{abstract}
M. Hovd ${ }^{1}$ S. Olaru ${ }^{2}$
${ }^{1}$ Department of Engineering Cybernetics, Norwegian University of Science and Technology, N-7491 Trondheim, Norway. E-mail: Morten.Hovd@itk.ntnu.no

${ }^{2}$ Automatic Control Department, CentraleSupelec, 3 rue Joliot Curie, 91192 France. E-mail: sorin.olaru@centralesupelec.fr

Abstract

The calculation of piecewise quadratic (PWQ) Lyapunov functions is addressed in view of stability analysis of uncertain piecewise linear dynamics. As main contribution, the linear matrix inequality (LMI) approach proposed in Johansson and Rantzer (1998) for the stability analysis of PWL and PWA dynamics is extended to account for parametric uncertainty based on a improved relaxation technique. The results are applied for the analysis of a Phase Locked Loop (PLL) benchmark and the ability to guarantee a stability region in the parameter space well beyond the state of the art is demonstrated.
\end{abstract}

Keywords: Lyapunov stability; Piecewise linear/affine dynamics; Uncertain systems; Linear matrix inequalities

\section{Introduction}

Piecewise linear (PWL) and piecewise affine (PWA) systems are natural extensions from linear toward a non-linear framework Sontag (1981) in control theory and appear often in control applications when piecewise linear components, such as saturation, relays or stick-slip friction are encountered Di Bernardo et al. (2007). Linear predictive control with constraints is also known to result in PWA closed-loop dynamics Bemporad et al. (2002), and in the wider literature, references can be found to related models such as for example PWA slab systems Rodrigues and Boyd (2005) or PWA systems derived by function approximation using hinging hyperplanes Breiman (1993). Recent work on hybrid systems has given further emphasis to PWA systems as an important class of models able to describe switching in the system dynamics and have been demonstrated to be equivalent with alternative model- ing frameworks Heemels et al. (2001).

PWA systems have been studied intensively, and pose challenging analysis problems Johansson (2003) that are often computationally demanding due to the inherent complexity of the state partition.

For PWA systems, the use of a same quadratic Lyapunov candidate function for all local system dynamics is often imposing a overconservative structural constraint and show not to be the appropriate tool for stability analysis. Starting from this observation, the use of PWQ Lyapunov functions calculated using an LMI approach was introduced by Johansson and Rantzer Johansson and Rantzer (1998). Although their definition uses local descriptions of the dynamics, the LMI formulations of the stability criteria were designed to hold globally. The conservatism of the initial PWQ approach was ameliorated in Rantzer and Johansson (2000) by the introduction of relaxations to the LMI formulation. These relaxations are such that the LMI 
formulation still ensures that the Lyapunov function stability criteria hold within the region of validity for each system dynamic, while relaxing the stability criteria outside that region. The same relaxation principles have been adapted to the discrete-time particularities Feng (2002); Ferrari-Trecate et al. (2002) and to Input to State Stability (ISS)-type of constructions Lazar (2006). In recent works, the conservatism of the PWQ approach is further reduced by introducing improved relaxations and allowing for sub-partitioning of the initial state space partition Hovd and Olaru (2013) or by the use of cone copositive PWQ Lyapunov functions Iervolino et al. (2015).

In a parallel line of development, LMI approaches have also been developed for investigating stability of uncertain linear systems, for both linear time-invariant (LTI) systems (see, e.g. Oliveira et al. (2008); Oliveira and Peres (2005) and references therein) and for linear time-varying (LTV) systems Daafouz and Bernussou (2001).

The aim of the present paper is to address the robust stability of piecewise linear ( $P W L$ ) discrete time systems, were the dynamics in each partition of the state space are described by time-varying dynamics. The proposed technique is putting together the LMI conditions for PWA dynamics with those for uncertain dynamics. We provide the proof of robust stability for the proposed LMI conditions and apply the obtained results for a challenging problem, the stability of a PLL systems Akre et al. (2012). Finally we point to the generalization of the theoretic results to PWA systems with linear time-varying parameter dependence.

Notations: The positive orthant of a finite dimensional vector space $\mathbb{R}^{n}$ is denoting the subset $\left\{x \in \mathbb{R}^{n} \| x_{i} \geq 0, \forall 0 \leq i \leq n\right\}$. For a matrix $A \in \mathbb{R}^{n \times m}$, the notation $A_{i j}$ denotes the element on line $i$, column $j$. If the matrix is square $(n=m)$ then $\operatorname{diag}(A)$ denotes the diagonal matrix having $A_{i i}=\operatorname{diag}(A)_{i i}, \forall 1 \leq i \leq n$ and $\operatorname{diag}(A)_{i j}=0, \forall i \neq j$.

\section{System dynamics and quadratic forms}

\subsection{Piecewise linear dynamics}

Consider a state space (or some compact convex subset thereof) $X$ of dimension $n$, partitioned into nonoverlapping regions $X_{i}, i \in \mathcal{I}$ such that

$$
X=\cup_{i \in \mathcal{I}} X_{i} .
$$

where the index set $\mathcal{I}$ is a bounded subset of $\mathbb{N}$. The system dynamics are governed by:

$$
\begin{aligned}
x(t+1) & =f_{\text {pwl }}(x(t)) \\
& =A\left(\lambda_{i}(t)\right) x(t) \text { for } x(t) \in X_{i}
\end{aligned}
$$

where $t$ indicates discrete time. For each index $i$, the vector of parameters $\lambda_{i} \in \mathbb{R}^{n_{i}}$ is described by a function of time:

$$
\lambda_{i}: \mathbb{R} \rightarrow D_{i} \subset \mathbb{R}^{n_{i}}
$$

where $n_{i}$ represents the local number of unknown parameters for each state space region $X_{i}$.

Both the structure of the functions $A(),. \lambda_{i}($.$) and$ the topology of the sets $X_{i}, D_{i}$ offer a large number of degrees of freedom for the description of dynamics (2). In the present paper we are interested in linear (possibly) time-varying matrix, $A\left(\lambda_{i}(t)\right)$ :

$$
A\left(\lambda_{i}(t)\right)=\sum_{k=1}^{n_{i}} \lambda_{i}^{k}(t) A_{i}^{k}
$$

where $\lambda_{i}^{k}$ denotes the $k$-th element of the vector $\lambda_{i}$ and $X_{i} \subset \mathbb{R}^{n}$ are polyhedral regions satisfying $\operatorname{int}\left(X_{i} \cap\right.$ $\left.X_{j}=\emptyset\right)$.

Over each region $X_{i}$, the linear dependence of the transition matrix on the vector of parameters (4) leads to a polytopic difference inclusion. The system dynamics are bounded by a combination of $n_{i}$ extreme realizations of the transition matrix and the scalars $\lambda_{i}^{k}(t)$ represent the linear combination coefficients. If the value of $\lambda_{i}^{k}$ is fixed but unknown $\lambda_{i}^{k}(t)=\lambda_{i}^{k}(0)$, then we are dealing with a $P W L$ with parametric uncertainty while in the case when the value of $\lambda_{i}^{k}(t)$ may change between timesteps, as indicated by the dependence in $t$, we are dealing with a time-varying $P W L$ system. Note that this second case, the parameter variation can take place even when the state trajectory remains in the same partition $X_{i}$ of the state space. Up to this point, there is no particular structural condition on the topology of the sets $D_{i} \subset \mathbb{R}^{n_{i}}$.

A particular case of linear interpolation is the convex combination for which the co-domain $D_{i}$ in (3) is a simplex in the positive orthant of 'parameter space':

$$
S_{i}=\left\{\lambda_{i} \in \mathbb{R}^{n_{i}} \mid \lambda_{i}^{k} \geq 0 ; \sum_{k=1}^{n_{i}} \lambda_{i}^{k}=1\right\}
$$

The convexity of this set represents a valuable property which will be exploited in the stability analysis. The key element in this endeavor will be the construction of a Lyapunov function. In the present paper we concentrate on the stability analysis via the PWQ candidate function: $V: \mathbb{R}^{n} \times \mathbb{R}^{n_{i}} \rightarrow \mathbb{R}_{+}$defined as:

$$
V\left(x, \lambda_{i}\right)=x^{T} \mathcal{P}_{i}\left(\lambda_{i}\right) x \quad \text { for } \quad x \in X_{i} .
$$

with $\mathcal{P}_{i}: \mathbb{R}^{n_{i}} \rightarrow \mathbb{R}^{n \times n}$ and advocate the use, for each partition $i$, of the specific form

$$
\mathcal{P}_{i}\left(\lambda_{i}\right)=\sum_{k=1}^{n_{i}} \lambda_{i}^{k} P_{i}^{k}
$$


Each particular matrix $P_{i}^{k}$ is defined as a Lyapunov function valid for the extreme dynamics associated to the extreme transition matrix $A_{i}^{k}$.

Before presenting the main result let us introduce a well-posedness hypothesis and two supplementary notations.

Hypothesis 1 The set $X$ is invariant with respect to $(2)$, i.e. $\forall x(t) \in X_{i}$ we have $f_{\text {pwl }}(x(t)) \in X$.

In relationship with the set of states with feasible transitions let us denote:

$$
X_{i j}^{k}=\left\{x \in X_{i} \mid A_{k}^{i} x_{t} \in X_{j}\right\}
$$

and

$$
X_{i j}=\operatorname{co}\left\{\cup_{k} X_{i j}^{k}\right\}
$$

where co denotes the convex hull.

\subsection{Quadratic forms over simplices}

An instrumental result on the quadratic forms defined over simplex type of domains is stated in the next Lemma. It will be subsequently employed for relaxations in the stability analysis context in the next section.

Lemma 1 Consider a quadratic function:

$$
q(\lambda): \mathbb{R}^{n_{i}} \rightarrow \mathbb{R}
$$

with $q(\lambda)=\lambda^{T} Q \lambda$ and $Q=Q^{T}$.

Let $S$ denote the simplex in the positive orthant of $\mathbb{R}^{n_{i}}$ as in (5) and $\mathcal{V}(S)$ its set of $n_{i}$ vertices.

If for any pair $\left(\lambda_{i}, \lambda_{j}\right) \in \mathcal{V}(S) \times \mathcal{V}(S)$ with $\lambda_{i} \neq \lambda_{j}$ there exists a scalar $0<\alpha<1$ such that:

$$
q\left(\alpha \lambda_{i}+(1-\alpha) \lambda_{j}\right) \geq \alpha^{2} Q_{i i}+(1-\alpha)^{2} Q_{j j}
$$

then

$$
q(\lambda) \geq \lambda^{T} \operatorname{diag}(Q) \lambda, \forall \lambda \in S
$$

Proof: First let us introduce the quadratic form

$$
\tilde{q}(\lambda)=q(\lambda)-\lambda^{T} \operatorname{diag}(Q) \lambda
$$

and describe it explicitly as $\tilde{q}(\lambda)=\lambda^{T} \tilde{Q} \lambda$ via a symmetric matrix $\tilde{Q}$. We observe that the diagonal elements of both quadratic matrices describing the quadratic form $\tilde{q}($.$) coincide and by consequence$ $\operatorname{diag}(\tilde{Q})=0$.

This structural property implies that by construction $\tilde{q}\left(\lambda_{i}\right)=0$ for any vertex $\lambda_{i} \in \mathcal{V}(S)$ as long as for such a vector $\lambda_{i}$ only one element is 1 while the other elements are zero.
Let us now concentrate on the points expressed as linear combinations of two vertices of the simplex:

$$
\begin{aligned}
\lambda_{i j}(\alpha)=\alpha \lambda_{i}+ & (1-\alpha) \lambda_{j}, \\
& \left(\lambda_{i}, \lambda_{j}\right) \in \mathcal{V}(S) \times \mathcal{V}(S)
\end{aligned}
$$

with $\alpha$ a scalar denoting the interpolation coefficient. The value of the quadratic function $\tilde{q}(\lambda)$ is determined by the off-diagonal elements of the symmetric matrix $\tilde{Q}$ and more specifically

$$
\tilde{q}\left(\lambda_{i j}(\alpha)\right)=2 \tilde{Q}_{i j} \alpha(1-\alpha)
$$

Following the inequality (10), there exist at least one scalar $0<\alpha<1$ such that $\tilde{q}\left(\lambda_{i j}(\alpha)\right) \geq 0$ which indicates that $Q_{i j}>0$. The existence of such positive function values for all pairs of vertices $\left(\lambda_{i}, \lambda_{j}\right) \in$ $\mathcal{V}(S) \times \mathcal{V}(S)$ implies that all off-diagonal elements of $\tilde{Q}$ are positive.

Since for $\lambda \in S$ all elements of the vector are nonnegative (as interior point of a simplex in the positive orthant - (5)), the quadratic function $\tilde{q}($.$) is non-$ negative over the simplex $S$ and finally:

$$
\begin{aligned}
& \tilde{q}(\lambda) \geq 0, \forall \lambda \in S \\
& q(\lambda) \geq \lambda^{T} \operatorname{diag}(Q) \lambda, \forall \lambda \in S
\end{aligned}
$$

thus proving the statement.

Practically, the Lemma 1 states that if for each onedimensional edge connecting two vertices of the simplex, one point can be found where the value of the quadratic function is larger than the value corresponding to a quadratic interpolation between the function values at the corresponding vertices, then the function values at all non-extreme points in the simplex is larger than the value obtained from quadratic interpolation between values at simplex vertices. This can be extended to the positive quadratic forms as formally stated in the next result.

Corollary 1 Consider a quadratic function defined over a simplex in positive orthant of $S \subset \mathbb{R}^{n}$

$$
q(\lambda): \mathbb{R}^{n} \rightarrow \mathbb{R}
$$

with $q(\lambda)=\lambda^{T} Q \lambda$ and $Q=Q^{T}$.

If for any pair $\left(\lambda_{i}, \lambda_{j}\right) \in \mathcal{V}(S) \times \mathcal{V}(S)$ with $\lambda_{i} \neq \lambda_{j}$ there exists a scalar $0<\alpha<1$ such that:

- $q(\lambda) \geq 0, \forall \lambda \in \mathcal{V}(S)$

- $\exists \alpha \in(0,1)$ such that

$$
q\left(\alpha \lambda_{i}+(1-\alpha) \lambda_{j}\right) \geq \alpha^{2} Q_{i i}+(1-\alpha)^{2} Q_{j j}
$$

then

$$
q(\lambda) \geq 0, \forall \lambda \in S
$$


Proof: The proof is immediate by observing that the positivity over the vertices of the simplex $S$ leads to the positivity of the diagonal terms, $Q_{i i}, \forall 1 \leq i \leq n$. Then inequality (14) is then a direct implication of the Lemma 1.

These elements will allow the use of a quadratic form which is guaranteed to be positive over a simplex but to allow negative values outside this region by means of a concave type of structure.

\section{PWQ Stability}

\subsection{Stability criteria for nominal PWL time-invariant systems}

The stability results for the nominal PWL timeinvariant (PWLTI) systems will be reviewed first. Nominal is understood here in the sense that $n_{i}=1$, for all $i$ in the state space partition (4)-(5).

Proposition 1 The nominal system (2)-(4) is piecewise quadratically stable provided the Lyapunov function (6) verifies

$$
\begin{aligned}
& V(x)>0, \forall x \neq 0 \\
& V(x)-V\left(f_{\text {pwlti }}(x)\right)>0, \forall x \neq 0 .
\end{aligned}
$$

For the nominal PWL LTI systems there is no parametric uncertainty, and we thus have $\mathcal{P}_{i}=P_{i}$ as a constant matrix and $A\left(\lambda_{i}(t)\right)=A_{i}(1)=A_{i}$. Following Rantzer and Johansson (2000) and Feng (2002) relaxations can be introduced and stability guaranteed if $P_{i}=P_{i}^{T}$ and

$$
\begin{array}{rll}
P_{i}-F_{i} & > & 0, \forall i \\
A_{i}^{T} P_{j} A_{i}-P_{i}+G_{i j} & < & 0, \\
\forall(i, j) & \text { s.t. } & X_{i j} \neq \emptyset
\end{array}
$$

The equations (16) and (17) represent relaxation of the classical quadratic LMI conditions, obtained upon additional constraints on the auxiliary matrices $F_{i}$ and $G_{i j}$ :

$$
\begin{aligned}
x^{T} F_{i} x & >0, \forall x \in X_{i} \\
x^{T} G_{i j} x & >0, \forall x \in X_{i j} .
\end{aligned}
$$

\subsection{Stability criteria for PWL LTV systems}

In the Linear Time Varying case, for each region $X_{i} \subset$ $\mathbb{R}^{n}$, the parameters evolve in a simplex $\lambda_{i} \in S_{i}$. We will denote by $\mathcal{V}_{i}$ the set of indexes of vertices for this simplex, its cardinal being à priori known:

$$
\mathcal{V}_{i}=\left\{k \in \mathbb{N} \mid 1<k \leq n_{i}\right\}
$$

Imposing (16) to each vertex $k \in \mathcal{V}_{i}$ and (17) to each pair of vertices $(k, m) \in \mathcal{V}_{i} \times \mathcal{V}_{j}$ one obtains

$$
\begin{aligned}
P_{i}^{k} & -F_{i}^{k}>0, \forall k, i \\
\left(A_{i}^{k}\right)^{T} P_{j}^{m}\left(A_{i}^{k}\right) & -P_{i}^{k}+G_{i j}^{k}<0 \\
\forall(k, m) & \in \mathcal{V}_{i} \times \mathcal{V}_{j} ; \\
\forall(i, j) & \in\left\{i, j \mid X_{i j} \neq \emptyset\right\}
\end{aligned}
$$

Define $\lambda_{i}^{\bar{k} l}$ to be the value of the parameter vector on the midpoint on each one-dimensional edge of the simplex $S_{i}$ :

$$
\lambda_{i}^{\overline{k l}}=0.5\left(\lambda_{i}^{k}+\lambda_{i}^{l}\right)
$$

For a simplex region, there are $\frac{n_{i} \text { ! }}{2\left(n_{i}-2\right) !}$ such points where elements $\lambda_{i}^{k}$ and $\lambda_{i}^{l}$ of $\lambda_{i}$ equal 0.5 and all other elements are zero. Likewise, define the corresponding dynamics $A_{i}^{\bar{k} l}=A\left(\lambda_{i}^{\bar{k} l}\right)$, and the corresponding value $\mathcal{P}_{i}^{\bar{k} l}$ of the Lyapunov function (c.f. (7)). We then impose the additional condition

$$
\begin{gathered}
0.5\left[\left(A_{i}^{k}\right)^{T} P_{j}^{m}\left(A_{i}^{k}\right)+\left(A_{i}^{l}\right)^{T} P_{j}^{m}\left(A_{i}^{l}\right)\right] \\
-\left(A_{i}^{k l}\right)^{T} P_{j}^{m}\left(A_{i}^{\overline{k l}}\right)-H_{m}^{k l}>0 \\
\forall k, l \in \mathcal{V}_{i} ; m \in \mathcal{V}_{j} ; \forall(i, j) \in\left\{i, j \mid X_{i j} \neq \emptyset\right\}
\end{gathered}
$$

where $H_{m}^{\overline{k l}}$ defines some relaxation function that is positive on $X_{i j}$.

Theorem 1 If the matrices $P_{i}^{k}, F_{i}^{k}, G_{i j}^{k}, H_{m}^{\overline{k l}}$ can be found that fulfill (20) - (23), the system described by (2) is asymptotically stable.

Proof: Equation (20) ensures that each Lyapunov function is positive over the partition for which it is valid (and the weighted sum with positive parameters in (7) is hence also positive over the same partition). Equation (21) ensures that the Lyapunov function decreases for all extreme realizations of the parameters $\lambda_{k}^{i}$ defining the dynamics within partition $X_{i}$ (i.e., for all vertices of the parameter simplex), and for all values of $\lambda_{k}^{j}$ defining the Lyapunov function $\mathcal{P}_{j}$. Note that since $(21)$ is linear in $\lambda_{k}^{j}$, fulfilling the criterion at all vertices of the parameter simplex also ensures fulfilling the criterion at internal points. In contrast, (21) is quadratic in $\lambda_{k}^{i}$, and we need additional criteria to ensure that the Lyapunov function decreases for intermediate values of $\lambda_{k}^{i}$.

Equation (23) ensures that the decrease in the Lyapunov function according to (21) is greater at the midpoints of each (one-dimensional) edge connecting two vertices of the parameter simplex, than at the corresponding vertices. The theorem then follows from the Lemma above.

In some cases, the parameter variation may affect the dynamics only in some directions in the state space, 
i.e., the difference $\Delta A_{k l}=A_{k}^{i}-A_{l}^{i}$ may not be a full rank matrix. In such cases, there will be a subspace $\Omega \subset \mathbb{R}^{n}$ of the state space where the terms in (23) that depend on $P_{j}^{m}$ sum to zero. If the constraints on the relaxation $H_{m}^{k l}$ are such that the relaxation has to be positive over parts of $\Omega$, it will be impossible to fulfill (23) while at the same time fulfilling the constraints on the relaxation.

On the other hand, we observe that for states $x \in \Omega$, the requirement for decrease of the value of the Lyapunov function is fulfilled by the conditions that ensure decrease of the Lyapunov function at the vertices of the parameter simplex. One needs therefore impose the condition (23) only in the directions in state space where the dynamics is affected by the parameter uncertainty. This leads to the modified condition

$$
\begin{aligned}
& v_{f}^{T}\left[0.5\left(\left(A_{k}^{i}\right)^{T} P_{j}^{m}\left(A_{k}^{i}\right)+\left(A_{l}^{i}\right)^{T} P_{j}^{m}\left(A_{l}^{i}\right)\right)\right. \\
& \left.-\left(A_{k l}^{i}\right)^{T} P_{j}^{m}\left(A_{k l}^{i}\right)-H_{m}^{k l}\right] v_{f}>0, \forall k, l ; \forall m
\end{aligned}
$$

where $v_{f}$ defines a basis for the subspace $\Omega^{\perp}$, and can readily be obtained from a singular value decomposition of $\Delta A_{k l}$.

\subsection{Stability criteria for uncertain PWL time-invariant systems}

In this case, the cardinal of extreme realizations in the convex combination (4)-(5) can be $n_{i}>1$ but a restriction is placed on $\lambda_{i}^{k}(t)=\lambda_{i}^{k}(0)$ which is constant but unknown. Clearly, the conditions (20) - (23) guaranteeing the stability of the LPV case are sufficient for the stability of uncertain LTI systems.

As a final remark, even if in the original definitions of the dynamics (2)-(3)-(4), the sets $D_{i}$ are not convex, they can be approximated by a union of such convex sets (principally by simplices as in (5)).

\section{Example}

A PWL system related to a Phase Locked Loop system Akre et al. (2012) will be considered for the numerical illustration. The dynamics are defined by

$$
\begin{aligned}
x(t+1) & =A_{i} x(t) \text { for } x(t) \in X_{i}, i \in\{1,2\} \\
X_{1} & =\left\{x \in \mathbb{R}^{2} \mid\left[\begin{array}{ll}
1 & 0
\end{array}\right] x \geq 0\right\} \\
X_{2} & =\left\{x \in \mathbb{R}^{2} \mid\left[\begin{array}{ll}
1 & 0
\end{array}\right] x<0\right\}
\end{aligned}
$$

and the matrices

$$
\begin{aligned}
& A_{1}=\left[\begin{array}{rr}
2-\left[\begin{array}{rrr}
1 & 0
\end{array}\right] \lambda_{1} & -1-\left[\begin{array}{rr}
1 & 0
\end{array}\right] \lambda_{1} \\
1 & 0
\end{array}\right] ; \\
& A_{2}=\left[\begin{array}{rrr}
2 & -1-\left[\begin{array}{rr}
1 & 1
\end{array}\right] \lambda_{2} \\
1 & 0
\end{array}\right] .
\end{aligned}
$$

This system may be stable or unstable depending on the values of the parameters $\lambda_{1}$ and $\lambda_{2}$. The stability of this system was studied in Akre et al. (2010), and stability regions based both on algebraic criteria and based on simulation were presented. The problem with analyzing stability of PWL systems using simulation, is that stability of trajectories may depend on the initial state, and the domain of attraction for the unstable dynamics may be very small. This probably explains the 'rough' shape of the stable region found using simulation in Akre et al. (2010).

The parameter $\lambda_{1} \in \mathbb{R}^{2}$ will be considered to take values in the simplex $D_{1}=\operatorname{co}\{(0,0),(2,-2),(4,0)\}^{1}$ and for this region the stability is to be analyzed. The same domain of variation will be considered for the vector of parameters $\lambda_{2}$. Thus the stability analysis has to be done for $\lambda_{1}, \lambda_{2} \in \operatorname{co}\{(0,0),(2,-2),(4,0)\}$. This simplex is subdivided into smaller simplices for stability analysis, since the system is known not to be stable for all parameter values in the original simplex. In the LMI-based stability analysis, sub-partitioning of the original state space partition is used, as proposed in Hovd and Olaru (2013). Each of the original state space partitions are sub-divided into $4-32$ partitions, as required to prove stability. If stability is not proven when using 32 sub-partitions for each of the original partitions in the state space, the simplex in the $\left(k_{1}, k_{2}\right)$ space under study is either further sub-divided, or it is concluded that stability cannot be proven for that simplex.

Exploring the $\left(\lambda_{1}, \lambda_{2}\right)$ space, the stable area shown in Fig. 1 is found.

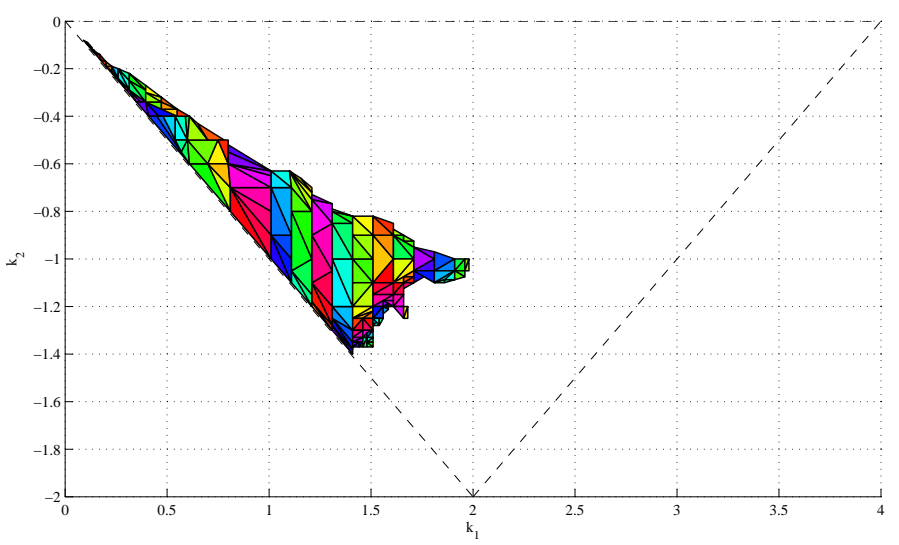

Figure 1: Stable area in $\left(k_{1}, k_{2}\right)$ space found using proposed LMI technique.

The stable area shown in the figure is significantly larger than the one based on algebraic criteria in Akre et al. (2010), but smaller than the region found

\footnotetext{
${ }^{1}$ Here co represents the convex hull, as defined after (9)
} 
using (unreliable) simulation. Considering the somewhat irregular shape of the stable area in Fig. 1, it seems reasonable to expect that a somewhat larger region can be proven stable. However, that would require automated procedures for exploring the $\left(\lambda_{1}, \lambda_{2}\right)$ space.

It should be noted that the increase in the area for which the system is proven stable is achieved despite inherent conservatism in our approach, since we allow for time-varying parameter uncertainty whereas in the original description the parameters are uncertain but constant. Furthermore, the condition (23), requiring the decrease in the Lyapunov function along the edges of the parameter simplex to exceed the decrease at the vertices, is clearly conservative if the decrease of the Lyapunov function is large at the vertices.

\section{Conclusion}

New LMI-based stability criteria for robust stability of piecewise linear time-varying systems is proposed. Extension to piecewise affine time-varying systems is straight forward, using the lifting technique in Johansson and Rantzer (1998). The versatility of the results are illustrated using an example from literature.

\section{References}

Akre, J.-M., Juillard, J., Galayko, D., and Colinet, E. Synchronization analysis of networks of selfsampled all-digital phase-locked loops. IEEE Transactions on Circuits and Systems -I, 2012. 4:708-720. doi:10.1109/TCSI.2011.2169745.

Akre, J. M., Juillard, J., Olaru, S., and Galayko, D. Determination of the behavior of selfsampled digital phase-locked loops. In 53rd IEEE Int. MWSCAS. pages 1089-1092, 2010. doi:10.1109/MWSCAS.2010.5548840.

Bemporad, A., Morari, M., Dua, V., and Pistikopoulos, E. N. The explicit linear quadratic regulator for constrained systems. Automatica, 2002. 38:3-20. doi:10.1016/S0005-1098(01)00174-1.

Breiman, L. Hinging hyperplanes for regression, classification, and function approximation. Information Theory, IEEE Transactions on, 1993. 39(3):9991013. doi:10.1109/18.256506.

Daafouz, J. and Bernussou, J. Parameter dependent lyapunov functions for discrete time systems with time varying parametric uncertainties. Systems \& Control Letters, 2001. pages 355-359. doi:10.1016/S0167-6911(01)00118-9.
Di Bernardo, M., Budd, C., Champneys, A., and Kowalczyk, P. Piecewise smooth dynamical systems: theory and applications Applied mathematical sciences, vol. 163. 2007. doi:10.1007/978-1-84628-7084.

Feng, G. Stability analysis of piecewise discrete-time linear systems. IEEE Trans. Autom. Contr., 2002. 47:1108-1112. doi:10.1109/TAC.2002.800666.

Ferrari-Trecate, G., Cuzzola, F. A., Mignone, D., and Morari, M. Analysis of discrete-time piecewise affine and hybrid systems. Automatica, 2002. 38:21392146. doi:10.1016/S0005-1098(02)00142-5.

Heemels, W., De Schutter, B., and Bemporad, A. Equivalence of hybrid dynamical models. Automatica, 2001. 37(7):1085-1091. doi:10.1016/S00051098(01)00059-0.

Hovd, M. and Olaru, S. Relaxing pwq lyapunov stability criteria for pwa systems. Automatica, $2013 . \quad 49(2): 667-670$. doi:10.1016/j.automatica.2012.10.013.

Iervolino, R., Vasca, F., and Iannelli, L. Conecopositive piecewise quadratic lyapunov functions for conewise linear systems. Automatic Control, IEEE Transactions on, 2015. 60(11):3077-3082. doi:10.1109/TAC.2015.2409933.

Johansson, M. Piecewise linear control systems: a computational approach. Springer Verlag, 2003. doi:10.1007/3-540-36801-9.

Johansson, M. and Rantzer, A. Computation of piecewise quadratic lyapunov functions for hybrid systems. IEEE Transactions on Automatic Control, 1998. 43:555-559. doi:10.1109/9.664157.

Lazar, M. Model predictive control of hybrid systems: Stability and robustness. Ph.D. thesis, Technische Universiteit Eindhoven, Department of Electrical Engineering, 2006.

Oliveira, R., de Oliveira, M. C., and Peres, P. Convergent lmi relaxations for robust analysis of uncertain linear systems using lifted polynomial parameter-dependent lyapunov functions. Systems \& Control Letters, 2008. pages 680-689. doi:10.1016/j.sysconle.2008.01.006.

Oliveira, R. and Peres, P. Lmi conditions for robust stability analysis based on polynomially parameter-dependent lyapunov functions. Systems \& Control Letters, 2005. pages 52-61. doi:10.1016/j.sysconle.2005.05.003. 
Rantzer, A. and Johansson, M. Piecewise linear quadratic optimal control. IEEE Transactions on Automatic Control, 2000. 45:629-637. doi:10.1109/9.847100.

Rodrigues, L. and Boyd, S. Piecewise-affine state feedback for piecewise-affine slab systems using convex optimization. Systems \& Control Letters, 2005.
54(9):835-853. doi:10.1016/j.sysconle.2005.01.002.

Sontag, E. Nonlinear regulation: The piecewise linear approach. Automatic Control, IEEE Transactions on, 1981. 26(2):346-358. doi:10.1109/TAC.1981.1102596. 